\title{
20 Anos de Plano Real: uma análise da relação entre câmbio, inflação, taxa de juros e o lbovespa
}

Henrique Nogueira Santana

Doutorando em Administração - CEPEAD/UFMG

Coordenador Financeiro-Administrativo - SOFTRUCK BRASIL

hnsantana@hotmail.com

Sabrina Amélia de Lima e Silva

Doutoranda em Administração - CEPEAD/UFMG

Professora Substituta no Departamento de Estatística - Universidade Federal de Minas Gerais

silva.saamelia@gmail.com

Bruno Pérez Ferreira

Doutor em Administração - CEPEAD/UFMG -Universidade Federal de Minas Gerais.

Professor Adjunto no Departamento de Administração da UFMG

brunoperez@ufmg.br

\section{Editor Científico: José Edson Lara}

Organização Comitê Científico

Double Blind Review pelo SEER/OJS

Recebido em 27.09.2017

Aprovado em 08.03.2018 


\section{Resumo}

Este artigo investigou a associação entre indicadores econômicos (taxas de juros, inflação e câmbio) com o mercado de ações brasileiro (lbovespa) no período dos 20 anos do Plano Real (jul./1994 a jun./2014), o qual foi contemplado por diversas eleições presidenciais e as crises de México, Rússia e Argentina, além da crise do subprime, em 2008. Utilizou-se o modelo de Vetor Autorregressivo (VAR), com análise das Funções de Resposta a Impulso (IRF), Análise de Decomposição das Variâncias (VDC) e Modelo de Correção de Erro (VECM). Os resultados mostram que há causalidade entre as variáveis econômicas e o lbovespa no sentido preditor do índice. Verificou-se que o modelo com VECM tem maior poder regressivo para a série do Ibovespa quando comparado com o modelo VAR, mostrando que as séries analisadas são cointegradas e que há uma forte correlação negativa entre a taxa de câmbio e o Ibovespa

Palavras-Chave: Ibovespa; Plano Real; Taxa de câmbio; Taxa de inflação; Taxa de juros.

\section{Years of Real Plan: an analysis of the relationship between exchange rate, inflation, interest rate and the lbovespa}

\section{Abstract}

This article investigated the association between economic indicators (interest, inflation and exchange rates) with the Brazilian stock market (lbovespa) in the 20-year period of the Real Plan (jul./1994 to jun./2014), which was considered by several presidential elections and the crises of Mexico, Russia and Argentina, in addition to the subprime crisis, in 2008. The Vector Autoregressive (VAR) model was used, with analysis of Impulse Response Functions (IRF), Variance Decomposition Analysis (VDC) and Error Correction Model (VECM). The results show that there is causality between the economic variables and the lbovespa in the predictive sense of the index. It was verified that the model with VECM has greater regressive power for the lbovespa series when compared to the VAR model, showing that the series analyzed are cointegrated and that there is a strong negative correlation between the exchange rate and the lbovespa.

Key words: Ibovespa; Real plan; Exchange rate; Inflation rate; Interest rate.

\section{Años de Real: un análisis de la relación entre cambio, inflación, tasa de interés y el lbovespa}

\section{Resumen}

Este artículo investigó la asociación entre indicadores económicos (tasas de interés, inflación y cambio) con el mercado de acciones brasileño (lbovespa) en el período de los 20 años del Plan Real (jul./1994 a jun./2014), el cual fue contemplado por diversas 
elecciones presidenciales y las crisis de México, Rusia y Argentina, además de la crisis del subprime, en 2008. Se utilizó el modelo de Vecino Autorregresivo (VAR), con análisis de las Funciones de Respuesta a Impulso (IRF), Análisis de Descomposición de Varianza (VDC) y Modelo de Corrección de Errores (VECM). Los resultados muestran que hay causalidad entre las variables económicas y el lbovespa en el sentido de predicción del índice. Se verificó que el modelo con VECM tiene mayor poder regresivo para la serie del lbovespa cuando comparado con el modelo VAR, mostrando que las series analizadas son cointegradas y que hay una fuerte correlación negativa entre la tasa de cambio y el lbovespa.

Palabras clave: Ibovespa; Plan Real; Tasa de cambio; Tasa de inflación; Tasa de interés.

\section{Introdução}

No século passado, o desenvolvimento das potências econômicas mundiais teve sustentação no crescimento de seus mercados de capitais, de forma que o financiamento pela população às empresas privadas proporcionava alavancagem financeira barata quando comparada às taxas praticadas por financiamentos bancários, além de permitir independência da intervenção do governo. Segundo Grôppo (2004), o mercado de capitais é, para as empresas, a principal e mais eficiente via para captação de recursos privados e ainda pode significar uma distribuição do risco do empreendimento por toda a sociedade, ao contrário do que aconteceria nos casos de autofinanciamento, créditos bancários ou fomento estatal, caracterizando uma concentração do risco.

Sabe-se que em períodos de crise econômico-financeira, há uma movimentação intensa dos governos para tentar controlar a situação dos seus países. Essa movimentação é, em suma, baseada em intervenções na economia a fim de se controlar as taxas de juros, taxas de inflação, o câmbio, dentre outros indicadores. Diante disso, torna-se importante conhecer se as medidas econômicas adotadas por um governo podem influenciar a atividade das empresas.

Diversos são os estudos que abordam a relação entre os preços dos ativos no mercado de ações e indicadores macroeconômicos. BahmaniOskooe e Sohrabian (1992), Ajayi, Friedman e Mehdian (1998) e Nunes, Costa Junior e Seabra (2002) mostram que a taxa de câmbio é um indicador que apresenta forte causalidade com os ativos. Já Blanchard (1981) observa que medidas de expansão fiscal (flexibilização de impostos ou aumento de gastos públicos), por exemplo, geram uma pequena 
variação no mercado de ações, algumas vezes positivas, outras vezes negativas. Ao testar a hipótese proxy de Fama (1981) para a bolsa de valores de Nova lorque nos períodos 1954 a 1976 e 1977 a 1990, Balduzzi (1994) corrobora com os resultados de Fama (1981) de que há correlação negativa entre inflação e retorno das ações.

No caso do mercado brasileiro, segundo Grôppo (2006), no período compreendido entre jan/1995 e jul/2005, as taxas de juros e o câmbio impactaram o Ibovespa, porém o câmbio é a variável mais impactante. Júnior e Scherma (2005) mostraram, porém, uma perspectiva contrária, em que os valores do Ibovespa são potenciais preditores da série de câmbio no período entre jan/1995 e dez/2004. Já segundo Júnior e Higuchi (2008), ao analisarem o período entre jul/1994 e jun/2005, não foi possível perceber relação de causalidade estatisticamente significativa dos juros, câmbio e inflação com o lbovespa.

Percebe-se, então, que há grande divergência na literatura com relação ao sentido da causalidade existente entre os indicadores macroeconômicos e os preços dos ativos no mercado acionário. Alguns estudos salientam que o mercado de ações influencia o comportamento dos juros, câmbio e inflação, enquanto outros apontam uma relação no sentido inverso. Além disso, é ainda escassa a literatura que aborda esse assunto para o mercado brasileiro, ainda menos quando se trata de análises referentes a períodos tão longos quanto os 20 anos do Plano Real, o qual contou com diversas eleições presidenciais e, também, crises tão relevantes quanto foram as crises do México (1994-1995), da Rússia (1998), da Argentina (2001-2002) e a crise do Subprime de 2008.

Torna-se importante, então, aprofundar o debate sobre o tema a fim de proporcionar entendimento aos investidores externos e internos sobre melhor alocação dos recursos em seus portfólios de investimentos. Além disso, e ainda mais importante, ao passo que a expansão da capacidade de produção representa um indicador de sucesso e desenvolvimento das economias, faz-se necessário que a direção administrativa das empresas compreenda quando o mercado de ações estará favorável ou não às suas companhias conforme o cenário econômico do momento, incluindo vésperas de eleições e sob temores de crise.

Diante do exposto, esse artigo visa responder à seguinte pergunta de pesquisa: Os indicadores macroeconômicos de juros, inflação e câmbio influenciaram o 
comportamento do retorno do lbovespa nos 20 anos após a implantação do Plano Real?

A importância desse período reside no fato de abranger mudanças consideráveis na economia brasileira, diversas eleições presidenciais e crises internacionais de impacto para o mercado doméstico. Então, o objetivo dessa pesquisa é contribuir para discussão de como as empresas de capital aberto e seus papéis na bolsa de valores foram atingidos pelas intervenções do governo na economia ao longo do Plano Real. Para tanto, buscou-se elaborar uma associação estatística de indicadores econômicos com o mercado de ações brasileiro no período dos 20 anos do Plano Real.

Esse entendimento torna-se importante por se tratar do mercado acionário de um país em desenvolvimento, como o Brasil, que está cada vez mais aberto a captar investimentos internacionais e que vem passando por um processo de modernização que se assemelha ao processo que muitos países desenvolvidos já se submeteram.

\section{Referencial Teórico}

\subsection{Revisão da Literatura}

Diversos são os estudos que tratam da relação dos indicadores macroeconômicos com os preços dos ativos no mercado de ações. É o caso de Blanchard (1981), Fama (1981), Chen, Roll e Ross (1986), Balduzzi (1994), Jones e Kaul (1996), Panetta (2002), Shamsuddin e Kim (2003), Campbell e Vuolteenaho (2004), Junior e Scherma (2005), Nunes, Costa Jr. e Meurer (2005), Grôppo (2006), Araújo e Bastos (2008) e Júnior e Higuchi (2008).

Em seu estudo, Chen, Roll e Ross (1986) apresentam os fundamentos da relação entre os indicadores de mercado e o retorno acionário, em que o mercado de ações sofre efeito das variações macroeconômicas, pois as empresas são afetadas em suas taxas de desconto, capacidade de gerar fluxos de caixas e capacidade de pagamento de dividendos. Para eles, o preço dos ativos depende da sua exposição às inúmeras variações dos índices do estado que descrevem a economia. A produção industrial e o prêmio de risco explicam significativamente o retorno esperado das ações e as mudanças na inflação esperada também explicam o retorno das ações, porém com menor significância. 
BahmaniOskooe e Sohrabian (1992), Ajayi, Friedman e Mehdian (1998) e Nunes, Costa Jr. e Seabra (2002) mostram que a taxa de câmbio é um indicador que apresenta forte causalidade com os ativos. No caso do mercado brasileiro, Grôppo (2006) constatou que as taxas de juros e o câmbio impactaram o lbovespa, porém o câmbio é a variável mais impactante, no período compreendido entre jan/1995 e jul/2005.

Júnior e Scherma (2005) mostraram que os valores do lbovespa são potenciais preditores da série de câmbio no período entre jan/1995 e dez/2004. Nunes, costa Jr. e Meurer (2005) chegaram a conclusões parecidas, de forma que a relação entre o setor externo e o lbovespa foi verificada através das taxas de câmbio e que as inovações no lbovespa podem explicar as variações na taxa de câmbio.

Segundo Fama (1981), a combinação da relação positiva entre retorno das ações e crescimento real da economia com a relação negativa entre inflação e crescimento real da economia resulta na relação negativa entre retorno das ações e inflação. Balduzzi (1994), ao testar a hipótese proxy de Fama (1981) para a bolsa de valores de Nova Yorque nos períodos 1954 a 1976 e 1977 a 1990, também conclui que há correlação negativa entre inflação e retorno das ações e que essa correlação negativa é alavancada pela taxa de juros.

Por sua vez, Campbell e Vuolteenaho (2004) verificaram que o nível de inflação explica $80 \%$ da série de variação das ações, porém seu estudo aborda a volatilidade de ações mal precificadas. Para eles, há evidências estatisticamente significativas de que essa volatilidade está relacionada com os efeitos da inflação sobre o crescimento dos dividendos pagos pelas ações. Já segundo Júnior e Higuchi (2008), ao analisarem o caso brasileiro no período pós Plano Real, entre julho de 1994 e junho de 2005, não foi possível perceber relação de causalidade estatisticamente significativa dos juros, câmbio e inflação com o índice lbovespa.

Essa rápida revisão da literatura mostra o quão divergente são os estudos sobre o tema. Tais divergências impedem que se faça previsões acerca de como um mercado de ações pode se comportar ao longo do tempo, diante de variações de políticas econômicas, sob pressões internacionais ou qualquer tipo de instabilidade econômico-financeira. 


\subsection{Os 20 anos de Plano Real}

O Plano Real começou a ser elaborado em 1993, durante o Governo Itamar Franco, pelo então Ministro da Fazenda, Fernando Henrique Cardoso, e sua equipe econômica. Segundo Andrezo e Lima (1999), esse era um plano de estabilização que pretendia controlar a inflação mediante a liberação da economia brasileira ao comércio mundial, especialmente às importações, com uma taxa de câmbio que apresentava elevada apreciação de nossa moeda frente ao dólar americano. Dividido em três fases, ajuste das contas públicas, implantação da Unidade Real de Valor (URV) e transformação da URV em Real, o Plano Real tornou circulante o Real no dia $1^{\circ}$ de julho de 1994, no final do mandato de Itamar Franco.

Conforme Andrezo e Lima (1999), o Plano Real veio à tona num ambiente econômico mundial de alta liquidez nos mercados financeiros desenvolvidos em decorrência de um favorável volume de comércio mundial. Então, de fato, segundo Lameira (2004), o tão sonhado controle inflacionário tornou-se concreto utilizando de uma ampla abertura ao comércio exterior, além de uma melhor integração do sistema financeiro aos mercados financeiros internacionais.

Segundo Rennó e Spanakos (2006), o crescimento econômico de países com economia emergente depende de capital estrangeiro. No Brasil, com o Plano Real não foi diferente. No relatório econômico da ANDIMA (1999), a âncora cambial gerava um elevado déficit em conta corrente (transações do país com o exterior) que haveria de ser financiado pela entrada de capitais estrangeiros, através de investimentos diretos e de curto prazo, que se aproveitavam das altas taxas internas de juros, impostas pela autoridade monetária para diminuir a liquidez observada na economia proporcionada pela grande oferta de recursos externos.

Segundo Lameira (2004), a oferta de recursos e a demanda crescente proporcionaram, no período de 1995 a 1998, o controle da inflação e um crescimento do PIB de 4,2\%, 2,7\% e 3,3\% nos anos de 1995, 1996 e 1997, respectivamente. Ainda segundo o autor, o sucesso do Plano Real estava acompanhado por um aumento da transparência da atuação do Banco Central, além do bem-sucedido estabelecimento de metas de inflação como objetivo da política monetária e da administração do câmbio através de regime flutuante a partir de 1998. 
Todo o cenário da economia nacional, desde o início do Plano Real, sofreu influência de crises econômicas em outros países e, também, dos processos eleitorais ocorridos nos 20 anos de trajetória. No caso da influência sofrida por crises em outras economias, ressalta-se as "Crises dos Emergentes", focando nas crises do México (1994-1995), Rússia (1998) e Argentina (2001-2002), além da crise do Subprime em 2008. Já sobre os processos eleitorais, cinco foram as disputas ocorridas nesses 20 anos: 1994, com a eleição de Fernando Henrique Cardoso; 1998, com a reeleição deste; 2002, com vitória do candidato da oposição, Luiz Inácio Lula; 2006, com a reeleição de Lula; e 2010 com vitória da candidata da situação, Dilma Rousseff.

\subsection{Crises dos emergentes e do Subprime}

Segundo Pinto, Vilela e Lima (2005), define-se crise financeira em um país quando há um ataque especulativo à sua moeda corrente, o que pode resultar numa significativa desvalorização da moeda local e, possivelmente, no não pagamento de suas dívidas. Segundo Aldrighi e Cardoso (2009), as crises financeiras são antecedidas por um período de valorização nos preços dos ativos. Essa valorização aumenta a vulnerabilidade financeira da economia ao passo que bancos e investidores afrouxam os critérios de avaliação de risco.

Já as crises cambiais, por sua vez, têm por característica, segundo Aldrighi e Cardoso (2009), a perda substancial de reservas internacionais do governo ou do banco central, que decorrem de uma reversão nas expectativas dos investidores sobre os rumos da taxa de câmbio no país e sobre sua capacidade de solvência. Para eles, a crise financeira pode, inclusive, desencadear uma crise cambial à medida que investidores e credores estrangeiros passam a liquidar seus ativos na economia em crise devido a uma correção sob suas expectativas. Uma vez desencadeadas, as crises financeira e cambial se reforçariam mutuamente.

Em seu estudo, ao fazerem uma comparação entre as crises financeira e cambial da América Latina e Leste Asiático, Aldrighi e Cardoso (2009), mostraram evidências de que as economias do Leste Asiático apresentavam mais vulnerabilidades quanto ao setor bancário. Já nas economias latino-americanas, as fragilidades estavam relacionadas aos desequilíbrios fiscais e às transações correntes 
do balanço de pagamentos. Essas características podem ser atribuídas tanto à crise da Rússia (1998), quanto às crises do México (1994-1995) e da Argentina (20012002).

Conforme Alberini e Boguszewski (2008), antes da crise do Subprime, em 2008, numa manobra de redução das taxas de juros a $1 \%$ ao ano, para viabilizar a recuperação dos mercados que estavam sofrendo com uma enorme desvalorização das ações de algumas empresas do setor tecnológico, o governo americano proporcionou uma retomada do mercado de trabalho e da renda, estimulando as famílias norte-americanas a aceitar o crédito farto para concretizar o sonho da casa própria. Porém, em muitos casos, o que se fazia era especulação com a possibilidade de alta nos preços. A partir daí o incremento da demanda por moradias concretizou os reflexos sobre as cotações dos imóveis, e as altas nos preços realimentavam esse processo.

Ainda segundo os autores, o mercado de hipotecas residenciais nos Estados Unidos era dominado pelo segmento prime, que contava com regras relativamente rígidas de avaliação de crédito e limites do valor a ser financiado. No entanto, existiam ainda outros dois segmentos de crédito, dentre as quais se encontrava o subprime, no qual era fornecido acesso a crédito aos mutuários com um histórico de crédito não tão bom, permitindo-Ihes a aquisição de imóveis próprios e a possibilidade de financiar outros gastos ou liquidar empréstimos e financiamentos.

Segundo Alberini e Boguszewski (2008), quando os preços dos imóveis começaram a cair em 2007, juntamente à alta dos juros, os lucros que eram obtidos de refinanciamentos dos empréstimos imobiliários acabaram devido a atrasos e inadimplência dos empréstimos imobiliários. Isso provocou queda nos preços que, juntamente à inadimplência, comprometeu o sistema bancário americano, restringindo a liquidez e atingindo o lado real da economia.

Com o cenário da crise formado, os Bancos Centrais Americano e Europeu agiram rapidamente, fornecendo liquidez aos mercados para evitar um colapso financeiro imediato. Nos Estados Unidos, o Banco Central iniciou uma agressiva redução das taxas de juros. De forma similar, o Brasil também teve sua economia atingida pela crise. Segundo Alberini e Boguszewski (2008), o primeiro impacto foi registrado pela Bolsa de Valores de São Paulo, que apresentou quedas de 10\% em um único dia, no segundo semestre de 2008. Além disso, as taxas de juros que 
estavam em constante redução apresentaram uma mudança de tendência. Essa elevação nos juros refletiu também na elevação das expectativas de inflação que, para o ano corrente, já ultrapassara o centro da meta fixada pelo Banco Central, gerando a valorização das commodities e o aumento nos preços dos alimentos.

\section{Metodologia}

Com base em dados secundários, o presente estudo tem uma abordagem quantitativa e foi conduzido como uma pesquisa descritiva, pois visa descobrir se há relações entre variáveis, pressupondo um relacionamento causal, mas sem se apegar à explicação das causas originárias dessa correlação.

Os dados usados foram coletados em base mensal do site do lpeadata e são: taxa de juros (SELIC), taxa de inflação (IPCA), taxa de câmbio (PTAX compra - fim do período) - indicadores da economia brasileira - e o lbovespa (fechamento) - índice representativo da Bolsa de Valores, Mercadorias e Futuros de São Paulo. O período de análise vai de julho de 1994 a junho de 2014, referindo-se, portanto, aos 20 anos do Plano Real e os testes foram executados no programa GRETL (Gnu Regression, Econometrics and Time-series Library).

\subsection{Modelo de Vetor Autorregressivo (VAR)}

A análise de causalidade entre as variáveis se deu a partir de um modelo de Vetor Autorregressivo (VAR) que, segundo Grôppo (2006), permite a análise do comportamento das variáveis em resposta a choques individuais, $n$ período à frente, a partir da Função de Resposta a Impulso (IRF). Esse modelo também permite que se faça inferências do poder explanatório de cada variável sobre as demais a partir da Análise de Decomposição das Variâncias (VDC).

Antes de se rodar o modelo VAR, a primeira etapa de uma análise econométrica de causalidade é a verificação da ordem de integração das séries de tempo estudadas, pois somente séries estacionárias são passíveis de estimação por regressão. A estacionariedade entre séries de tempo ocorre se elas forem integradas de ordem zero I( 0 ) ou se forem integradas de mesma ordem I(d). A estacionariedade 
das variáveis foi verificada segundo o teste de raiz unitária ADF (Dickey e Fuller Aumentado), que se dá a partir do teste de hipóteses com os coeficientes estimados do modelo representado pela equação 1 .

$$
\Delta Y_{t}=\alpha+\beta_{t}+\gamma Y_{t-1}+\sum_{i=1}^{p-1} \delta_{i} \Delta Y_{t-i}+\varepsilon_{t}
$$

Em que $\alpha$ representa $o$ intercepto, $\beta_{t}$ representa uma variável tendência, $\Delta Y_{t-1}$ representa os termos diferenciados defasados $\left(\Delta Y_{t-1}=Y_{t-1}-Y_{t-2} ; \Delta Y_{t-2}=Y_{t-2}-Y_{t-3}\right.$ etc. $)$ e $\varepsilon_{t}$ representa um erro de ruído branco.

No teste ADF, deve-se testar a hipótese nula $H_{0}: y=0$ contra a hipótese alternativa $H_{1}: Y<1$, utilizando o processo de estimação de mínimos quadrados ordinários, em que $Y=0$ faz com que $Y_{t}$ possa ter raiz unitária e ser uma série não estacionária e $|\gamma|<1$ faz com que $Y_{t}$ não tenha raiz unitária e seja uma série estacionária, descrita como um processo autorregressivo de ordem um, AR (1).

A metodologia VAR, segundo Grôppo (2006), independe da existência de um modelo estrutural econômico que retrate o relacionamento das variáveis envolvidas, deixando de lado a questão relativa à dependência ou independência dessas variáveis. Os modelos VAR, contanto, não podem ser usados para fazer inferências sobre a estrutura econômica.

Segundo Margarido (2000), a utilização dos modelos VAR exigem apenas a especificação do conjunto de variáveis que se acredita interagirem entre si dentro do sistema e a determinação do número de defasagens necessárias para captar a dinâmica dessa interação. De forma direta, o processo VAR pode ser representado pela equação 2 .

$$
B_{0} X_{t}=B_{1} X_{t-1}+B_{2} X_{t-2}+\cdots+B_{p} X_{t-p}+\varepsilon_{t}=\sum_{j=1}^{p} B_{j} X_{t-j}+\varepsilon_{t}
$$

Em que $X_{t}$ é um vetor $(n \times 1)$ das variáveis empregadas no modelo, $B_{0}$ é a matriz de relações contemporâneas, $B_{j}$ são matrizes $(n \times n)$ dos coeficientes que relacionam 
os valores defasados das variáveis com seus respectivos valores correntes e $\varepsilon_{t}$ representa um vetor $(n \times 1)$ de erros de ruído branco.

Sendo $X_{t}$ um processo estocástico estacionário com $n$ componentes, tomando um modelo de médias móveis e considerando que ele pode ser escrito em sua forma autorregressiva, o modelo passa a ser representada pela equação 3 .

$$
X_{t}=A_{0} \varepsilon_{t}+A_{1} \varepsilon_{t-1}+A_{2} \varepsilon_{t-2}+\cdots+A_{k} \varepsilon_{k-1}
$$

Em que $X_{t}$ é um vetor $(n \times 1)$ de $n$ séries temporais, $A_{k}$ são matrizes $(n \times n)$ de parâmetros e $A_{0}=I_{n}$.

Dado o modelo VAR, após determinada a ordem de defasagem $p$ é possível analisar a resposta aos choques através do método de Funções de Resposta a Impulso (IRF) aplicado à representação de médias móveis da equação 3, que ao ter seus coeficientes estimados permite descrever a resposta dinâmica dos componentes do sistema dada uma variação em qualquer um deles. Torna-se possível, portanto, ter boa visualização da elasticidade de impulso resposta. Considerando a equação $3 \mathrm{com}$ apenas duas variáveis, o modelo segue conforme equação 4 .

$$
\left[\begin{array}{l}
X_{1 t} \\
X_{2 t}
\end{array}\right]=\left[\begin{array}{ll}
1 & 0 \\
0 & 1
\end{array}\right]\left[\begin{array}{l}
\varepsilon_{1 t} \\
\varepsilon_{2 t}
\end{array}\right]+\left[\begin{array}{ll}
a_{11}^{1} & a_{12}^{1} \\
a_{21}^{1} & a_{22}^{1}
\end{array}\right]\left[\begin{array}{l}
\varepsilon_{1, t-1} \\
\varepsilon_{2, t-1}
\end{array}\right]+\left[\begin{array}{ll}
a_{11}^{2} & a_{12}^{2} \\
a_{21}^{2} & a_{22}^{2}
\end{array}\right]\left[\begin{array}{l}
\varepsilon_{1, t-2} \\
\varepsilon_{2, t-2}
\end{array}\right]+\cdots+\left[\begin{array}{ll}
a_{11}^{k} & a_{12}^{k} \\
a_{21}^{k} & a_{22}^{k}
\end{array}\right]\left[\begin{array}{l}
\varepsilon_{1, t-k} \\
\varepsilon_{2, t-k}
\end{array}\right]
$$

Nela, os elementos das matrizes $A_{i}$ medem a resposta do vetor $X_{t}$ a um choque unitário nos componentes dos vetores de inovação $\varepsilon_{i}$. Para que se verifique o efeito de um choque em apenas uma das variáveis sobre as demais variáveis do sistema, faz-se necessário alguns ajustes na matriz de variância e covariância, transformando-a em uma matriz diagonal e evitando que os choques contemporâneos aconteçam em mais de uma variável ou afetem mais de uma das variáveis, contaminando todo o sistema.

Outra análise aplicada ao modelo VAR é a Decomposição da Variância dos erros de previsão (VDC). Segundo Júnior e Scherma (2005), ela é capaz de mostrar qual fração do erro projetado para cada variável resulta do efeito de suas próprias inovações e qual fração do erro que resulta de inovações de outras variáveis. Segundo 
Grôppo (2004), supondo que a matriz de variância e covariância seja não singular, existe uma matriz $C$, triangular inferior e, também, não singular, representada na equação 5 , tal que $C C^{\prime}$ é igual à matriz de variância e covariância, expressa na equação 6 , em que $C^{\prime}$ é a matriz transposta de $C$.

$$
\begin{aligned}
& C=\left[\begin{array}{ll}
c_{11} & 0 \\
c_{21} & c_{22}
\end{array}\right] \\
& C C^{\prime}=\left[\begin{array}{cc}
c_{11}^{2} & \left(c_{11} c_{21}\right) \\
\left(c_{11} c_{21}\right) & \left(c_{21}^{2} c_{22}^{2}\right)
\end{array}\right]
\end{aligned}
$$

Em que os elementos da diagonal principal da equação 6 são somas de quadrados e permitem identificar a respectiva variância de cada choque unitário. Em outras palavras, é possível identificar a parcela da variância que corresponde ao próprio choque e a parcela da variância proveniente de choques das demais variáveis.

\subsection{Correção de Erro (VECM)}

Segundo Alexander (2005), o modelo de vetor autorregressivo, VAR, das diferenças de variáveis omite a especificação de equilíbrio se as variáveis são cointegradas. Além disso, apesar de não ser necessária, a cointegração, quando existe, é suficiente para caracterizar causalidade, o que significa que pontos de reversão em uma série precedem pontos de reversão na outra série.

O modelo torna-se bem especificado com relação ao equilíbrio quando se inclui termos defasados de desequilíbrio como variáveis explicativas. Este é, portanto, o Modelo de Correção de Erro (VECM), um modelo dinâmico das primeiras diferenças das variáveis, o qual apresenta uma análise de curto prazo das correlações dinâmicas. Nessa análise de curto prazo, identifica-se o termo de desequilíbrio $z$, que representa e corrige automaticamente os desvios do equilíbrio de longo prazo. Alexander (2005) ilustra o modelo VECM para duas ou mais séries cointegradas, conforme equação 7.

$$
\Delta y_{t}=\alpha_{0}+\boldsymbol{B}_{1} \Delta y_{t-1}+\boldsymbol{B}_{2} \Delta y_{t-2}+\cdots+\boldsymbol{B}_{p} \Delta y_{t-p}+\prod y_{t-1}+\varepsilon_{t}
$$

Em que a variável dependente é a primeira diferença e todas as equações têm as mesmas variáveis explicativas. 


\section{Apresentação e Discussão dos Resultados}

O resultado dos testes ADF rejeita a hipótese nula de raiz unitária para as variáveis IPCA e SELIC. Elas são, portanto, integradas de ordem zero, I (0), e estacionárias em nível. Porém, as variáveis PTAX e logaritmo natural do IBOVESPA, todas com constante e tendência, não têm a hipótese nula rejeitada, caracterizandose como autorregressivas de ordem um, AR (1). Para as primeiras diferenças dessas variáveis, o teste ADF rejeita a hipótese nula, caracterizando-as como estacionárias em sua primeira diferença e integradas de ordem um, I (1).

As variáveis componentes do modelo VAR são IPCA e SELIC, ambas em nível, e a primeiras diferenças de PTAX e IBOVESPA. Com o modelo VAR, é possível se conhecer a relação dinâmica entre o tempo de reação das respostas de umas variáveis frente aos choques nas outras variáveis, além da direção e duração dessas respostas. Para tanto, especifica-se primeiro o número de defasagens necessárias para captar a dinâmica das interações entre as variáveis.

As Tabelas 1 e 2 mostram as informações da regressão e os parâmetros de ajuste individual das variáveis na estimativa VAR do modelo com 1 defasagem. Para esse modelo, o tamanho da amostra foi de 238 e os critérios de informação AIC, BIC e HQC foram $-3,1845,-2,8343$ e $-3,0434$, respectivamente. A última coluna das tabelas, quando marcada com um asterisco, mostra que o ajuste da regressão ou as variáveis explicativas são significativas a um nível de significância de $5 \%$. Também foi feita a estimativa VAR para o modelo com 6 defasagens e os critérios de informação AIC, BIC e HQC foram -3,442, -1,9018 e -2,8210, respectivamente.

\section{Tabela 1}

Estimativa VAR (informações da regressão com 1 defasagem)

\begin{tabular}{|c|c|c|c|c|c|c|c|c|}
\hline $\begin{array}{l}\text { Variável } \\
\text { Depend. }\end{array}$ & $\begin{array}{l}\text { DP var. } \\
\text { depend. }\end{array}$ & $\begin{array}{c}\Sigma \text { quad. } \\
\text { Resid. }\end{array}$ & $\begin{array}{l}\text { EP da } \\
\text { regres. }\end{array}$ & $\mathbf{R}^{2}$ & $\mathbf{R}^{2}$ ajust. & $\begin{array}{c}\text { Est. } \\
F(5,232)\end{array}$ & p-valor & $\delta=5 \%$ \\
\hline ipca & 0,5293 & 26,2982 & 0,3367 & 0,6039 & 0,5954 & 70,7470 & 0,0000 & * \\
\hline selic & 0,7923 & 9,1335 & 0,1984 & 0,9386 & 0,9383 & 709,3617 & 0,0000 & * \\
\hline d_ptax & 0,1246 & 3,3802 & 0,1207 & 0,0812 & 0,0614 & 4,1019 & 0,0014 & * \\
\hline d_I_ibov & 0,0903 & 1,9156 & 0,0909 & 0,0090 & $-0,0123$ & 0,4234 & 0,8322 & \\
\hline
\end{tabular}


Fonte: Dados da pesquisa

Comparando os critérios de informação AIC, BIC e HQC, o modelo com 1 defasagem mostrou-se mais adequado, pois, apesar de apresentar o critério AIC menos negativo, tem os critérios BIC e HQC consideravelmente mais negativos do que o modelo com 6 defasagens.

\section{Tabela 2}

Estimativa VAR (parâmetros de ajuste individual das variáveis com 1 defasagem)

\begin{tabular}{|c|c|c|c|c|c|c|c|}
\hline $\begin{array}{c}\text { Variável } \\
\text { Dependente }\end{array}$ & $\begin{array}{c}\text { Variáveis } \\
\text { Explicativas }\end{array}$ & Coeficientes & $\begin{array}{c}\text { Erro } \\
\text { Padrão }\end{array}$ & $\begin{array}{c}\text { Estatística } \\
\mathrm{t}\end{array}$ & $\begin{array}{l}\text { Estatística } \\
F(1,232)\end{array}$ & p-valor & $\delta=5 \%$ \\
\hline \multirow{6}{*}{ Ipca } & Constante & $-0,093$ & 0,1296 & $-0,7204$ & & 0,4720 & \\
\hline & ipca_1 & 0,6511 & 0,0508 & 12,8300 & 164,5100 & 0,0000 & * \\
\hline & selic_1 & 0,1510 & 0,0530 & 2,8460 & 8,1025 & 0,0048 & * \\
\hline & d_ptax_1 & 0,1658 & 0,1882 & 0,8812 & 0,7765 & 0,3791 & \\
\hline & d_I_ibov_1 & 0,2501 & 0,2570 & 0,9731 & 0,9470 & 0,3315 & \\
\hline & Tendência & 0,0000 & 0,0000 & 1,1530 & & 0,2501 & \\
\hline \multirow{6}{*}{ selic } & Constante & 0,2921 & 0,0764 & 3,8230 & & 0,0002 & * \\
\hline & ipca_1 & 0,0468 & 0,0299 & 1,5630 & 2,4431 & 0,1194 & \\
\hline & selic_1 & 0,8624 & 0,0313 & 27,5800 & 760,8600 & 0,0000 & * \\
\hline & d_ptax_1 & $-0,0480$ & 0,1109 & $-0,4333$ & 0,1877 & 0,6652 & \\
\hline & d_l_ibov_1 & $-0,7653$ & 0,1514 & $-5,0530$ & 25,5360 & 0,0000 & * \\
\hline & Tendência & 0,0000 & 0,0000 & $-3,1640$ & & 0,0018 & * \\
\hline \multirow{6}{*}{ d_ptax } & Constante & 0,0314 & 0,0464 & 0,6753 & & 0,5002 & \\
\hline & ipca_1 & $-0,0369$ & 0,0182 & $-2,0270$ & 4,1103 & 0,0438 & * \\
\hline & selic_1 & 0,0109 & 0,0190 & 0,5718 & 0,3270 & 0,5680 & \\
\hline & d_ptax_1 & $-0,1867$ & 0,0675 & $-2,7680$ & 7,6601 & 0,0061 & * \\
\hline & d_l_ibov_1 & $-0,3250$ & 0,0921 & $-3,5280$ & 12,4450 & 0,0005 & * \\
\hline & Tendência & $-0,0001$ & 0,0002 & $-0,6108$ & & 0,5419 & \\
\hline \multirow{6}{*}{ d_I_ibov } & Constante & 0,0035 & 0,0350 & 0,1004 & & 0,9201 & \\
\hline & ipca_1 & 0,0051 & 0,0137 & 0,0694 & 0,1364 & 0,7122 & \\
\hline & selic_1 & 0,0024 & 0,0143 & 0,1712 & 0,0293 & 0,8642 & \\
\hline & d_ptax_1 & 0,0569 & 0,0508 & 1,1200 & 1,2543 & 0,2639 & \\
\hline & d_l_ibov_1 & 0,0502 & 0,0694 & 0,7235 & 0,5234 & 0,4701 & \\
\hline & Tendência & 0,0000 & 0,0001 & $-0,0779$ & & 0,9379 & \\
\hline
\end{tabular}

Fonte: Dados da pesquisa

Pela Tabela 1, percebe-se que as estimativas dos vetores autorregressivos baseadas em 1 defasagem apresentaram regressões significativas para as variáveis dependentes IPCA, SELIC e primeira diferença da PTAX. Isso que significa que a combinação entre todas as variáveis do sistema é explicativa das alterações em cada uma dessas variáveis dependentes. Fica claro, então, que a primeira diferença do 
logaritmo natural do IBOVESPA não pode ser explicada pela combinação das demais variáveis no período dos 20 anos de Plano Real.

Pela Tabela 2, vê-se que a própria variação do IPCA e da SELIC com 1 defasagem são explicativas do comportamento do IPCA. Para a SELIC, além da própria variação da SELIC e da primeira diferença do logaritmo natural do IBOVESPA com 1 defasagem, a regressão apontou também como significativas uma constante e uma tendência na explicação do comportamento da SELIC. Por último, para a primeira diferença da PTAX, a variação do IPCA, da própria primeira diferença da PTAX e da primeira diferença do logaritmo natural do IBOVESPA com 1 defasagem são significativas na explicação do comportamento da primeira diferença da PTAX.

Isso quer dizer que o IBOVESPA não explica as variações da taxa de inflação, mas explica as variações da taxa de juros e da taxa de câmbio. Porém, ele não é explicado por nenhuma das variáveis do sistema. A análise dos coeficientes permite perceber, também, que há uma correlação negativa entre SELIC e a primeira diferença do logaritmo natural do IBOVESPA e entre a primeira diferença da PTAX e a primeira diferença do logaritmo natural do IBOVESPA.

$\mathrm{Na}$ sequência, a função impulso resposta explicita a resposta da variável dependente no sistema VAR aos choques inesperados ocorridos nos termos de erro das variáveis explicativas. A Figura 1 mostra a intensidade e a duração das interferências causadas pelo incremento de um valor de um desvio padrão no termo de erro das variáveis explicativas sobre as variáveis dependentes. 


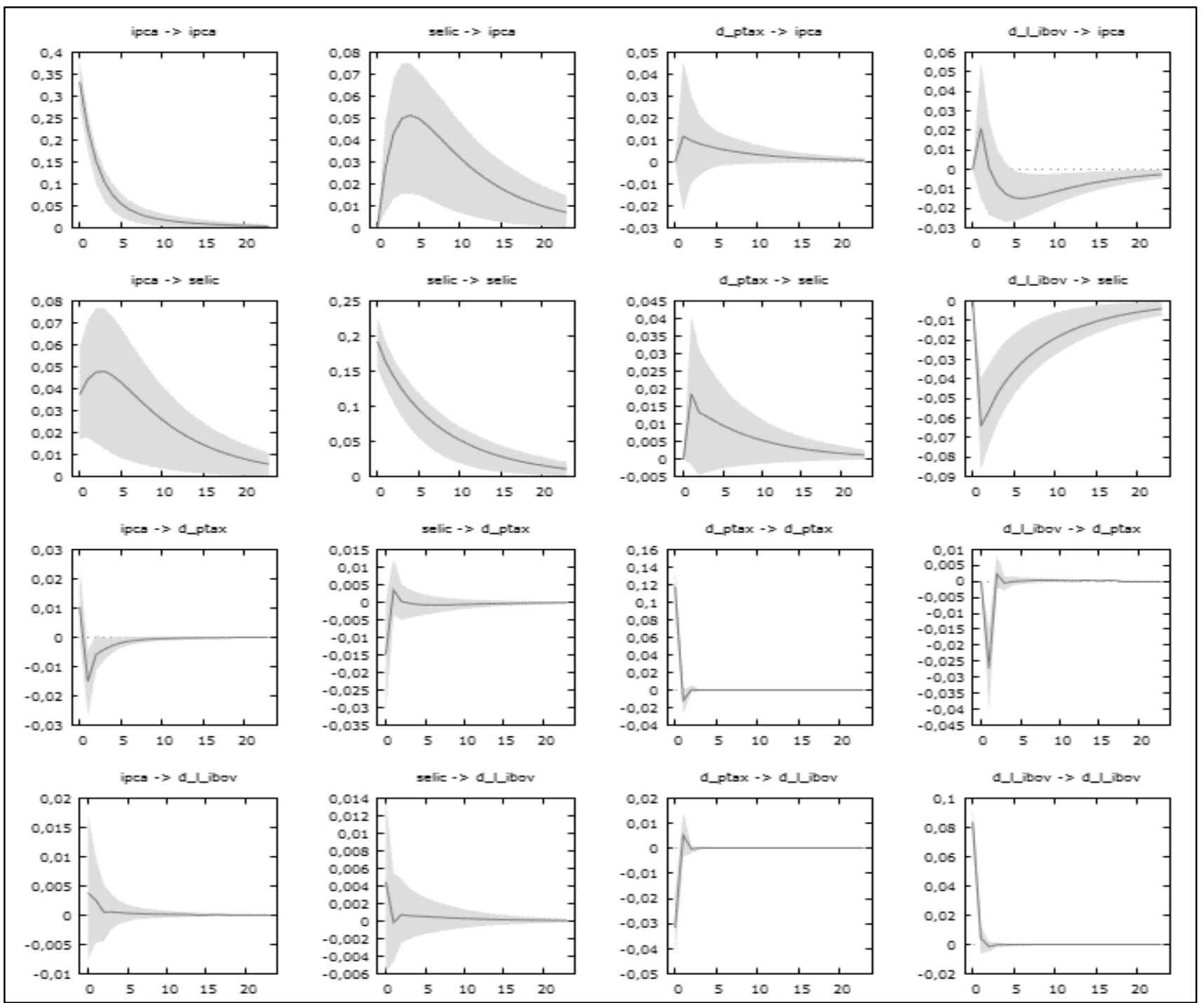

Figura 1

Gráficos da Função de Resposta a Impulso (IRF) para combinações entre IPCA, SELIC, Câmbio (ptax) e IBOVESPA

Fonte: Dados da pesquisa

É possível perceber que as variáveis dependentes que sofrem influências mais prolongadas devido aos choques nas variáveis explicativas são o IPCA (gráficos da primeira linha) e a SELIC (gráficos da segunda linha), pois a função demora vários períodos para reestabelecer o valor zero após o choque. Percebe-se que a maior intensidade da resposta ocorre quando os choques são provenientes da própria variável. No caso do IPCA, por exemplo, o incremento de um desvio padrão no termo de erro do IPCA tende a levar, no momento do choque, a um aumento de $33,24 \%$ do IPCA. 
Para a SELIC, um incremento de um desvio padrão no termo de erro da SELIC tende a levar, no momento do choque, a um aumento de $19,23 \%$ da SELIC. Já no caso da PTAX, um incremento de um desvio padrão no termo de erro da primeira diferença da PTAX tende a levar, no momento do choque, a um aumento de $11,78 \%$ da primeira diferença da PTAX. Porém, nesse último caso, a função tende a reestabelecer seu valor para zero já a partir do segundo período.

Essa mesma análise pode ser estendida para todas as combinações entre as variáveis do sistema. Nesse momento, não se pode deixar de observar que há uma correlação negativa entre SELIC e IBOVESPA e entre a primeira diferença da PTAX e a primeira diferença do logaritmo natural do IBOVESPA, conforme comprovado anteriormente, pois o incremento de um desvio padrão no termo de erro da primeira diferença do logaritmo natural do IBOVESPA tende a levar, nos períodos seguintes ao choque, a uma queda das variáveis dependentes SELIC e primeira diferença da PTAX.

A análise da Decomposição das Variâncias do Erro de Previsão (VDC) aplicada ao modelo VAR está resumida na Tabela 3 para os $1^{\circ}, 12^{\circ}$ e $24^{\circ}$ períodos posteriores. Através dela, é possível avaliar o poder explanatório de cada variável do modelo sobre as demais. Os resultados mostram que as próprias variáveis dependentes em análise são as principais explicativas de suas próprias variações.

\section{Tabela 3}

Decomposição das Variâncias do Erro de Previsão para o modelo VAR

\begin{tabular}{ccccccc}
\hline $\begin{array}{c}\text { Variável } \\
\text { Depend. }\end{array}$ & \multirow{2}{*}{ Período } & \multirow{2}{*}{$\begin{array}{c}\text { Erro } \\
\text { Padrão }\end{array}$} & \multicolumn{5}{c}{ Variáveis Explicativas } \\
\cline { 6 - 7 } ipca & 1 & 0,3324 & 100,00 & selic & d_ptax & d_I_ibov \\
& 12 & 0,4792 & 90,70 & - & - & - \\
& 24 & 0,4836 & 89,42 & 9,26 & 0,22 & 0,82 \\
\multirow{2}{*}{ selic } & 1 & 0,1959 & 3,62 & 96,38 & 0,23 & 0,98 \\
& 12 & 0,4248 & 9,86 & 80,63 & 0,63 & - \\
& 24 & 0,4370 & 10,32 & 80,12 & 0,63 & 8,93 \\
\hline \multirow{2}{*}{ d_ptax } & 1 & 0,1192 & 0,76 & 1,56 & 97,68 & - \\
& 12 & 0,1241 & 2,60 & 1,54 & 91,01 & 4,85 \\
& 24 & 0,1241 & 2,60 & 1,55 & 91,00 & 4,85 \\
\multirow{2}{*}{ d__ptax } & 1 & 0,0897 & 0,18 & 0,25 & 12,43 & 87,14 \\
& 12 & 0,0900 & 0,27 & 0,28 & 12,67 & 86,78 \\
& 24 & 0,0900 & 0,27 & 0,28 & 12,67 & 86,78 \\
\hline
\end{tabular}

Fonte: Dados da pesquisa 
Excetuando-se as próprias variáveis dependentes em cada caso, percebe-se que a SELIC é a variável explicativa mais representativa para a série do IPCA, assim como o IPCA é a variável explicativa mais representativa para a série da SELIC. A primeira diferença do logaritmo natural do IBOVESPA é a variável mais representativa para a primeira diferença da PTAX e a primeira diferença da PTAX é a mais explicativa da primeira diferença do logaritmo natural do IBOVESPA. Percebe-se, também, que as demais variáveis explicativas têm atuação crescente na explicação das variáveis dependentes até $012^{\circ}$ mês posterior e tende a alcançar uma estabilização a partir dele até o $24^{\circ}$ mês.

\subsection{Correção de Erro (VECM)}

Visto que o modelo VAR foi insuficiente para apresentar uma regressão significativa para a variável da primeira diferença do logaritmo natural do IBOVESPA, nesta seção será feita uma análise do modelo com Correção de Erro. Porém, serão utilizadas 2 defasagens, em vez de 1, pois a análise VECM consome uma defasagem para calcular a primeira diferença das variáveis de entrada.

As Tabelas 4 e 5 mostram, respectivamente, as informações da regressão e os parâmetros de ajuste individual das variáveis na análise VECM do modelo com 2 defasagens. Para esse modelo, o tamanho da amostra foi de 237 e os critérios de informação AIC, BIC e HQC foram -2,5737, -2,0469 e -2,3614, respectivamente.

\section{Tabela 4}

Estimativa VECM (informações da regressão com 2 defasagens)

\begin{tabular}{|c|c|c|c|c|c|}
\hline $\begin{array}{l}\text { Variável } \\
\text { Depend. }\end{array}$ & $\begin{array}{l}\text { DP var. } \\
\text { depend. }\end{array}$ & $\begin{array}{c}\Sigma \text { quad. } \\
\text { Resid. }\end{array}$ & $\begin{array}{l}\text { EP da } \\
\text { regres. }\end{array}$ & $\mathbf{R}^{2}$ & $\mathbf{R}^{2}$ ajust. \\
\hline d_ipca & 0,3668 & 30,1778 & 0,3614 & 0,0495 & 0,0289 \\
\hline d_selic & 0,2165 & 10,1441 & 0,2096 & 0,0833 & 0,0634 \\
\hline d_d_ptax & 0,1847 & 4,2482 & 0,1356 & 0,4724 & 0,461 \\
\hline d_d & 0,1263 & 2,3483 & 0,1008 & 0,3758 & 0,3623 \\
\hline
\end{tabular}

Fonte: Dados da pesquisa

Apesar de serem menos negativos do que os critérios de informação apresentados na análise VAR do modelo com 1 defasagem, esta análise apresentou-se mais adequada para a variável IBOVESPA, pois o $R^{2}$ e o $R^{2}$ ajustados são consideravelmente maiores e representam uma regressão com melhor ajuste, conforme Tabela 4. 
O ajuste proporcionado pelo modelo com Correção de Erro pode ser comprovado pela Tabela 5, a qual mostra, que todas as variáveis são explicativas da segunda diferença do logaritmo natural do IBOVESPA, inclusive ela mesma.

\section{Tabela 5}

Estimativa VECM (parâmetros de ajuste individual das variáveis com 2 defasagens)

\begin{tabular}{|c|c|c|c|c|c|c|}
\hline $\begin{array}{l}\text { Variável } \\
\text { Depend. }\end{array}$ & $\begin{array}{l}\text { Variáveis } \\
\text { Explic. }\end{array}$ & Coef. & $\begin{array}{c}\text { Erro } \\
\text { Padrão }\end{array}$ & Est. t & $p$-valor & $\delta=5 \%$ \\
\hline \multirow{6}{*}{ ipca } & Constante & $-0,0126$ & 0,0240 & $-0,5245$ & 0,6004 & \\
\hline & d ipca 1 & $-0,1070$ & 0,0658 & $-1,6250$ & 0,1056 & \\
\hline & d selic 1 & $-0,0785$ & 0,1164 & $-0,6746$ & 0,5006 & \\
\hline & d_d_ptax_1 & $-0,3875$ & 0,1673 & $-2,3160$ & 0,0215 & * \\
\hline & d_d_I_ibov_1 & $-0,0091$ & 0,2955 & $-0,0309$ & 0,9754 & \\
\hline & $\mathrm{EC} 1$ & 0,0036 & 0,0028 & 1,2660 & 0,2068 & \\
\hline \multirow{6}{*}{ selic } & Constante & $-0,0045$ & 0,0139 & $-0,3225$ & 0,7474 & \\
\hline & d_ipca_1 & 0,0778 & 0,0382 & 2,0390 & 0,0426 & * \\
\hline & d selic 1 & $-0,1252$ & 0,0675 & $-1,8550$ & 0,0649 & * \\
\hline & d_d_ptax_1 & 0,1230 & 0,0970 & 1,2680 & 0,2062 & \\
\hline & d_d_l_ibov_1 & 0,1479 & 0,1713 & 0,8632 & 0,3889 & \\
\hline & $\mathrm{EC} 1-$ & $-0,0054$ & 0,0016 & $-3,313$ & 0,0011 & * \\
\hline \multirow{6}{*}{ d_ptax } & Constante & 0,0093 & 0,0090 & 1,0360 & 0,3012 & \\
\hline & d_ipca_1 & $-0,0162$ & 0,0247 & $-0,6556$ & 0,5127 & \\
\hline & d_selic_1 & $-0,1046$ & 0,0437 & $-2,3940$ & 0,0175 & * \\
\hline & d d d ptax 1 & $-0,4255$ & 0,0628 & $-6,7780$ & 0,0000 & * \\
\hline & d_d_l_ibov_1 & 0,2624 & 0,1109 & 2,3670 & 0,0188 & * \\
\hline & $\mathrm{EC} 1^{-}$ & $-0,0060$ & 0,0010 & $-5,7470$ & 0,0000 & * \\
\hline \multirow{6}{*}{ d_l-ibov } & Constante & 0,0089 & 0,0067 & 1,3290 & 0,1850 & \\
\hline & d_ipca_1 & 0,0418 & 0,0184 & 2,2750 & 0,0238 & * \\
\hline & d_selic_1 & 0,0739 & 0,0325 & 2,2750 & 0,0238 & * \\
\hline & d d d ptax 1 & 0,2307 & 0,0467 & 4,9420 & 0,0000 & * \\
\hline & d_d_libov_1 & $-0,1559$ & 0,0824 & $-1,8910$ & 0,0598 & * \\
\hline & $\mathrm{EC}-\overline{-}$ & $-0,0045$ & 0,0008 & $-5,7340$ & 0,0000 & * \\
\hline
\end{tabular}

Fonte: Dados da pesquisa

Esse modelo constata, ainda, uma forte correlação negativa entre as segundas diferenças da PTAX e do logaritmo natural do IBOVESPA. Percebe-se, então, que esse conjunto de séries temporais são cointegradas. Além disso, a Tabela 6 mostra a matriz de covariâncias das equações cruzadas, a qual possui determinante zero.

\section{Tabela 6}

Matriz de covariâncias das equações cruzadas do modelo VECM com 2 defasagens

\begin{tabular}{lrrrr}
\hline & ipca & \multicolumn{1}{c}{ selic } & d_ptax & \multicolumn{1}{c}{ d_I_ibov } \\
\hline ipca & 0,1273 & 0,0118 & $-0,0018$ & 0,0052 \\
selic & 0,0118 & 0,0428 & $-0,0069$ & 0,0035 \\
d_ptax & $-0,0018$ & $-0,0069$ & 0,0179 & $-0,0065$ \\
d__ibov & 0,0052 & 0,0035 & $-0,0065$ & 0,0100 \\
\hline
\end{tabular}

Fonte: Dados da pesquisa 
A Figura 2 mostra os gráficos com o impacto da função impulso resposta (IRF) de cada variável explicativa do sistema sobre a variável dependente no modelo VECM, para 24 períodos à frente. Vê-se que todas as variáveis alcançam estabilização frente aos choques inesperados ocorridos nos termos de erro das variáveis explicativas.

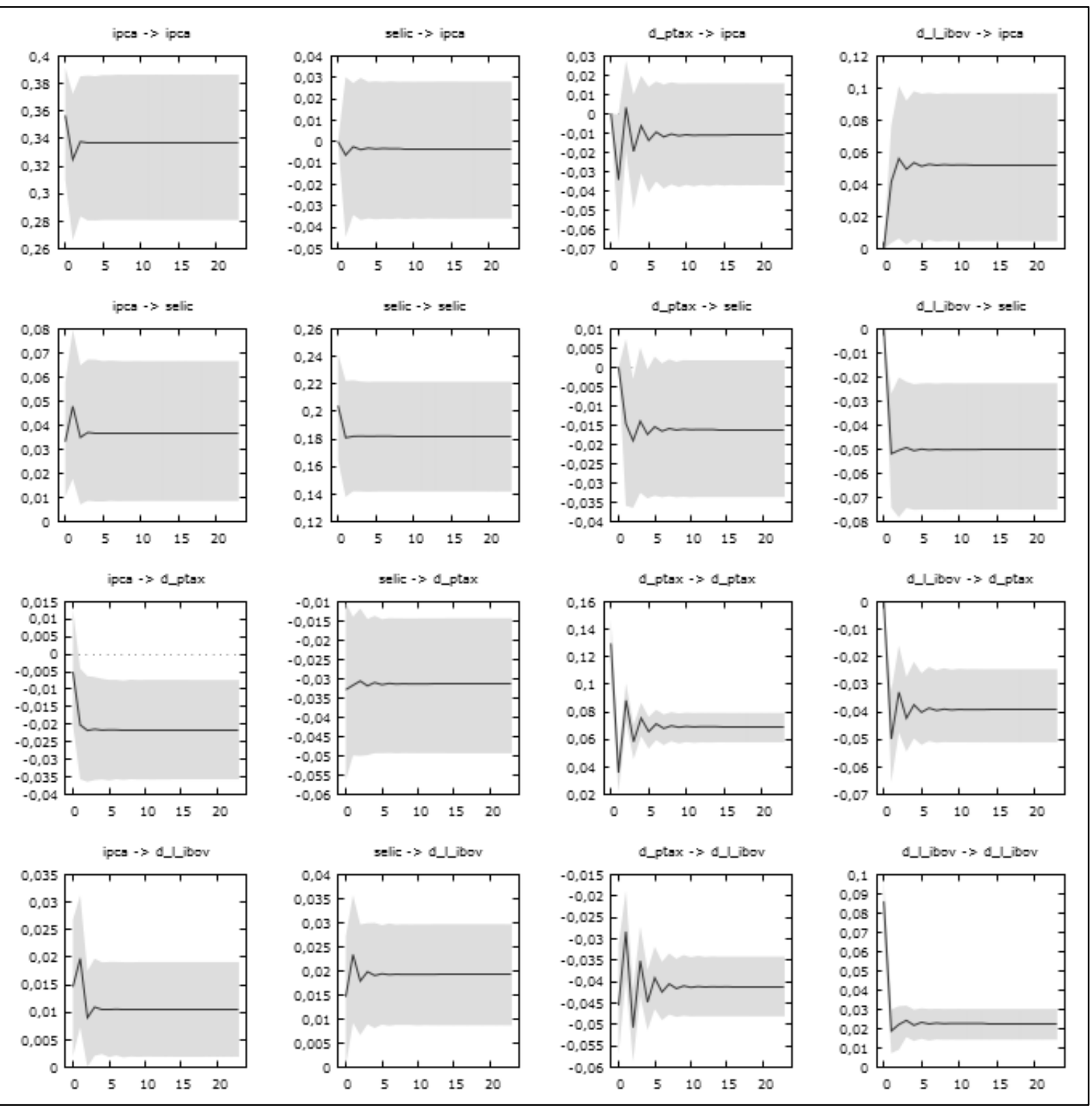

Figura 2

Gráfico da Função de Resposta a Impulso (IRF) para o modelo VECM Fonte: Dados da pesquisa

Por último, a análise da Decomposição das Variâncias do Erro de Previsão (VDC) para um horizonte de 24 períodos à frente permite melhor entendimento do 
poder explanatório de cada variável do modelo sobre as demais. Na Tabela 7, os resultados da VDC para o modelo com Correção de Erro, assim como para o VAR, mostram que as próprias variáveis dependentes em análise são as principais explicativas de suas próprias variações. Porém, para as primeiras diferenças da PTAX e do logaritmo natural do IBOVESPA, percebe-se que elas são explicadas com maior intensidade pelas demais variáveis do sistema, principalmente entre elas mesmas.

\section{Tabela 7}

Decomposição das Variâncias do Erro de Previsão para o modelo VECM

\begin{tabular}{ccccccc}
\hline $\begin{array}{c}\text { Variável } \\
\text { Depend. }\end{array}$ & \multirow{2}{*}{ Período } & \multirow{2}{*}{$\begin{array}{c}\text { Erro } \\
\text { Padrão }\end{array}$} & ipca & selic & d_ptax & d_I_ibov \\
\hline \multirow{2}{*}{ ipca } & 1 & 0,3568 & 100,00 & - & - & - \\
& 12 & 1,1846 & 97,72 & 0,01 & 0,18 & 2,08 \\
& 24 & 1,6743 & 97,64 & 0,01 & 0,14 & 2,21 \\
\hline \multirow{2}{*}{ selic } & 1 & 0,2068 & 2,57 & 97,42 & - & - \\
& 12 & 0,6732 & 3,70 & 89,55 & 0,63 & 6,12 \\
& 24 & 0,9488 & 3,65 & 89,25 & 0,66 & 6,43 \\
\hline \multirow{2}{*}{ d_ptax } & 1 & 0,1338 & 0,14 & 6,01 & 93,85 & - \\
& 12 & 0,3205 & 4,95 & 11,54 & 66,43 & 17,08 \\
& 24 & 0,4427 & 5,44 & 12,05 & 64,15 & 18,35 \\
\hline \multirow{2}{*}{ d__ptax } & 1 & 0,0995 & 2,15 & 2,16 & 20,96 & 74,73 \\
& 12 & 0,1990 & 4,28 & 11,22 & 51,71 & 32,79 \\
& 24 & 0,2684 & 4,21 & 12,37 & 56,73 & 26,69 \\
\hline
\end{tabular}

Fonte: Dados da pesquisa

A análise da Tabela 7 permite concluir que, ao final dos 24 períodos à frente, a primeira diferença do logaritmo natural do IBOVESPA é explicativa de $18,35 \%$ da primeira diferença da PTAX e que $56,73 \%$ da primeira diferença do logaritmo natural do IBOVESPA é explicado pela primeira diferença da PTAX.

\section{Considerações Finais}

Este estudo se propôs a analisar a relação estatística entre algumas variáveis macroeconômicas e o índice IBOVESPA no período dos 20 anos do Plano Real (julho de 1994 a junho de 2014). Para tanto, foi utilizado o enfoque multivariado VAR. Pretendia-se investigar se os indicadores econômicos têm influência sobre o preço dos ativos na Bolsa de Valores brasileira e se há, também, influência no sentido oposto. 
Verificou-se, após a análise do modelo VAR com as variáveis IPCA, SELIC, primeira diferença da PTAX e primeira diferença do logaritmo natural do IBOVESPA, que não há causalidade entre as variáveis econômicas e o IBOVESPA no sentido preditor do IBOVESPA. Esse resultado corrobora com o encontrado por Júnior e Higuchi (2008). O modelo mostra, também, que nem o próprio IBOVESPA é preditor de sua série de retornos. Porém, foi possível perceber que o IBOVESPA é uma variável estatisticamente explicativa da SELIC e da PTAX, o que contrapõe os resultados alcançados por Grôppo (2006), em que as taxas de juros e o câmbio impactaram o IBOVESPA. Há, entretanto, uma confirmação do que foi encontrado por Júnior e Scherma (2005) e Nunes, Costa Júnior e Meurer (2005), em que as inovações no IBOVESPA podem explicar as variações na taxa de câmbio.

O poder explicativo estatístico do IBOVESPA sobre a SELIC e a PTAX se dá segundo uma correlação negativa, o que está conforme o encontrado por Balduzzi (1994) para a bolsa de valores de Nova Yorque nos períodos 1954 a 1976 e 1977 a 1990, que também concluiu que a taxa de juros contribui fortemente para que haja uma correlação negativa entre inflação e retorno das ações.

Para se investigar a resposta das variáveis dependentes aos choques inesperados ocorridos nos termos de erro das variáveis explicativas, foi feito o teste IRF e constatou-se que as variáveis dependentes que sofrem influências mais prolongadas devido aos choques nas variáveis explicativas são o IPCA e a SELIC. Além disso, para todas as variáveis do sistema, percebeu-se que a maior intensidade da resposta ocorre quando os choques são provenientes da própria variável em análise. $O$ teste IRF confirmou a correlação estatística negativa entre SELIC e IBOVESPA e entre PTAX e IBOVESPA, pois o incremento de um desvio padrão no termo de erro da primeira diferença do logaritmo natural do IBOVESPA causou uma queda das variáveis dependentes SELIC e primeira diferença da PTAX.

Os resultados da análise da Decomposição das Variâncias do Erro de Previsão (VDC) se apresentam compatíveis aos obtidos através do teste IRF, o que permite perceber que as próprias variáveis dependentes em análise são as principais explicativas de suas próprias variações. O sistema de variáveis estudado apresenta relação estatisticamente significativa, porém, elas são numericamente fracas, o que quer dizer que ao nível de significância considerado, 5\%, foi possível perceber consistência nas regressões das séries das variáveis dependentes, mas o máximo 
poder explicativo encontrado foi de $12,67 \%$ da PTAX sobre o IBOVESPA, as quais não apresentaram correlação estatisticamente significativas.

Dada a insuficiência do modelo VAR em estabelecer relações estatísticas das demais variáveis com a primeira diferença do logaritmo natural do IBOVESPA, realizou-se a análise com Correção de Erro e constatou-se que as variáveis são cointegradas e que, nesse caso, o modelo VECM foi melhor representativo de relações estatísticas fortes entre as variáveis do sistema e o IBOVESPA. Verificou-se forte correlação negativa entre as primeiras diferenças da PTAX e do logaritmo natural do IBOVESPA, além de se comprovar que o IBOVESPA é explicado também pelo IPCA e pela SELIC.

Sugere-se que estudos alternativos em cima da mesma base de dados possam ser realizados, tais como realizar as mesmas análises em períodos com durações diversas, permitindo melhor entendimento sobre o comportamento das variáveis nas vésperas das eleições e das crises citadas ao longo do trabalho.

\section{Referências}

Ajayi, R. A., Friedman, J., \& Mehdian, S. M. (1998). On the relationship between stock returns and exchange rates: test of Granger causality. Global Finance Journal, 2(9), 241-251.

Alberini, D. V., \& Boguszewski, L. D. (2008). Por dentro do subprime: a crise imobiliária americana e seus impactos na economia brasileira. Vitrine da Conjuntura, 1(2), 1 10.

Aldrighi, D. M., \& Cardoso, A. D. (2009). Crises cambiais e financeira: uma comparação entre América Latina e Leste Asiático. Economia e Sociedade, 18(1), $61-117$.

Alexander, C. (2005). Modelos de Mercado: Um Guia para a Análise de Informações Financeiras. São Paulo: Saraiva.

Andima. (Dezembro 1999). Relatório econômico - Sistema financeiro no Mercosul: uma análise comparativa. Rio de Janeiro.

Andrezo, A. F., \& Lima, I. S. (1999). Mercado financeiro: aspectos históricos e conceituais. São Paulo: Pioneira.

Araújo, E., \& Bastos, F. A. S. (2008). Relações entre retornos acionários, juros, atividade econômica e inflação: evidências para a América Latina. BBR-Brazilian Business Review, 5(1), 51-72. 
Bahmanioskooee, M., \& Sohrabian, A. A. (1992). Stock prices and the effective exchange rate of the dollar. Applied Economics, 24(4), 459-464.

Balduzzi, P. (1994). Stock returns, inflation, and the 'proxy hypothesis': A new look at the data. Economics Letters, 48(1), 47-53.

Blanchard, O. (1981). J. Output, the stock market, and interest rates. The American Economic Review, 71(1), 132-143.

Chen, N. F., Roll, R., \& Ross, S. A. (1986). Economic forces and the stock market. Journal of Business, 59(3), 383-403.

Fama, E. F. (1981). Stock returns, real activity, inflation and money. The American Economic Review, 71(4), 545-565.

Grôppo, G. S. (2004). Causalidade das variáveis macroeconômicas sobre o lbovespa. (Dissertação de Mestrado, Universidade de São Paulo, SP, Brasil).

Grôppo, G. S. (2006). Relação dinâmica entre Ibovespa e variáveis de política monetária. Revista de Administração de Empresas, 46(1), 72-85.

Jones, C. M., \& Kaul, G. (1996). Oil and the stock markets. The Journal of Finance, 51(2), 463-491.

Júnior, T. P., \& Higuchi, R. H. (2008). Variáveis macroeconômicas e o IBOVESPA: um estudo da relação de causalidade. Revista Eletrônica de Administração, 14(2), 296315.

Júnior, T. P., \& Scherma, F. R. (2005). Um estudo da influência entre o dólar e o IBOVESPA no período 1999-2003. Revista Eletrônica de Gestão Organizacional, 3(1), 18-25.

Lameira, V. J. (2004). Uma revisão sobre a economia brasileira e o mercado financeiro após o Plano Real: as mudanças e a evolução do mercado de capitais entre 1995 e 2002. Revista Contabilidade e Finanças, 15(35), 96-110.

Margarido, M. A. (2000). Transmissão de preços agrícolas internacionais sobre preços agrícolas domésticos: o caso do Brasil. Tese de doutorado, Universidade de São Paulo, SP, Brasil.

Nunes, M. S., Costa Júnior, N. C. A., \& Meurer, R. (2005). A relação entre o mercado de ações e as variáveis macroeconômicas: uma análise econométrica para o Brasil. Revista Brasileira de Economia, 59(4), 585-607.

Nunes, M. S., Costa Junior, N. C. A., \& Seabra, F. (2002). Cointegração e causalidade entre variáveis macroeconômicas, "risco Brasil" e os retornos no mercado de ações brasileiro. Anais do Encontro de Economia Região Sul, Florianópolis, SC, Brasil, 5.

Panetta, F. (2002). The stability of the relation between the stock market and macroeconomic forces. Review of Banking. Finance and Monetary Economics, 31(3), 417-450. 
Pinto, B. J. M., Vilela, T. M. M., \& Lima, U. S. M. (2005). A crise financeira Russa. Economics Bulletin, 28(3).

Rennó, L., \& Spanakos, A. P. (2006). Fundamentos da economia, mercado financeiro e intenção de voto: As eleições presidenciais brasileiras de 1994, 1998 e 2002. Revista de Ciências Sociais, 49(1), 11-40.

Shamsuddin, A. F. M., \& Kim, J. H. (2003). Integration and interdependence of stock and foreign exchange markets: an Australian perspective. Journal of International Financial 